\title{
One-term Approximation for Normal Distribution Function
}

\author{
Omar Eidous*, Samar Al-Salman
}

Department of Statistics, Yarmouk University, Jordan

Copyright $\mathrm{C} 2016$ by authors, all rights reserved. Authors agree that this article remains permanently open access under the terms of the Creative Commons Attribution License 4.0 International License

\begin{abstract}
This paper presents a one-term approximation to the cumulative normal distribution functions. The absolute maximum error of the proposed approximation is 0.0018 less than 0.003 of Polya's approximation. Comparisons between the proposed approximation and the different approximations with one-term that stated in the literature are given.
\end{abstract}

Keywords Cumulative Distribution Function, Normal Distribution, Approximation

\section{Introduction}

Normal distribution is considered as one of the most important distribution functions in statistics because it is simple to handle analytically. That is, it reasonably describes many phenomena that occur in nature. It is well known that the cumulative normal distribution function does not have closed form representation. If a random variable $X$ is normally distributed with mean $\mu=0$ and variance $\sigma^{2}=1$ then its probability density function is given by

$$
f(x)=\frac{1}{\sqrt{2 \pi}} e^{-\frac{x^{2}}{2}},-\infty<x<\infty .
$$

The cumulative normal distribution function is

$$
\Phi(x)=\frac{1}{\sqrt{2 \pi}} \int_{-\infty}^{x} e^{-\frac{t^{2}}{2}} d t .
$$

Unfortunately, there is no closed-form solution available for the above integral and its value is usually found from the tables of the cumulative normal distribution for different values of $x$. A number of approximate functions for a cumulative normal distribution function have been reported in the research community (See for example, Aludaat and Alodat [1]; Johnson et al. [2]; Bailey [3] and Polya [4]). Let $\Phi^{*}(x)=\frac{1}{\sqrt{2 \pi}} \int_{0}^{x} e^{-\frac{t^{2}}{2}} d t$ then

$$
\begin{gathered}
\Phi(x)=0.5+\frac{1}{\sqrt{2 \pi}} \int_{0}^{x} e^{-\frac{t^{2}}{2}} d t \\
=0.5+\Phi^{*}(x), x \geq 0
\end{gathered}
$$

and since $\Phi(x)=1-\Phi(-x)$ for $x \geq 0$, then the problem of approximation $\Phi(x)$ is equivalent to the problem of approximation $\Phi^{*}(x)$.

This paper proposed a simple approximation with one-term for $\Phi(x), x \geq 0$. The idea of this proposed approximation is similar to that of Polya (1945) and Aludaat and Alodat [1]. However, the absolute maximum error of the proposed approximation is less than that of Aludaat and Alodat [1] and Polya [4]. For $x \geq 0$, the Polya's approximation for $\Phi(x)$ is,

$$
\Phi_{1}(x)=0.5\left(1+\sqrt{1-e^{-\frac{2}{\pi} x^{2}}}\right)
$$

and that of Aludaat and Alodat [1] is,

$$
\Phi_{2}(x)=0.5\left(1+\sqrt{1-e^{-\sqrt{\frac{\pi}{8}} x^{2}}}\right) .
$$

The maximum error of $\Phi_{1}(x)$ is 0.003 while it is 0.002 for $\Phi_{2}(x)$. The simplicity of the above two approximations come from the one-term to calculate $\Phi(x)$. The one-term approximation of Aludaat and Alodat [1] has absolute maximum error 0.002 less than 0.003 of Polya's one-term approximation. Another one-term approximation for $\Phi(x)$ is given by Bowling et al. [5], which is given by

$$
\Phi_{3}(x)=\frac{1}{1+e^{-1.702 x}} .
$$

The maximum error of $\Phi_{3}(x)$ is 0.0095 . Lin [6] gave the following formula to approximate $\Phi(x)$,

$$
\Phi_{4}(x)=\frac{1}{1+e^{-4 \pi x /(9-x)}} .
$$


The maximum error of $\Phi_{4}(x)$ is 0.0067 . There are many others approximations for $\Phi(x)$ that used more than one-term and offer more accurate approximations (see Johnson et al. [2] and Yerukala and Boiroju [7]).

\section{The Proposed Approximation}

Our main goal is to approximate

$$
\Phi(x)=\frac{1}{\sqrt{2 \pi}} \int_{-\infty}^{x} e^{-\frac{t^{2}}{2}} d t, x \geq 0 .
$$

According to Johnson et al. [2], the Ploya's approximation represents an upper bound for $\Phi(x)$, i.e.,

$$
\Phi(x) \leq 0.5\left(1+\sqrt{1-e^{-\frac{2}{\pi} x^{2}}}\right)
$$

Aludaat and Alodat [1] tried to improve the upper bound of $\Phi(x)$ by proving that

$$
\sqrt{1-e^{-\sqrt{\frac{\pi}{8} x^{2}}}} \leq \sqrt{1-e^{-\frac{2}{\pi} x^{2}}}
$$

However, the validity of the above inequality does not necessary implies that

$$
\Phi(x) \leq 0.5\left(1+\sqrt{1-e^{-\sqrt{\frac{\pi}{8}} x^{2}}}\right) .
$$

See also the numerical results of Table 1. Therefore, the claiming of Aludaat and Alodat [1] that their approximation is sharper than the Polya's upper bound approximation is not adequate. But it is still true that their approximation has an absolute maximum error less than that of Polya's one. In other words and to approximate $\Phi(x)$, the quantity

$$
0.5\left(1+\sqrt{1-e^{-\sqrt{\frac{\pi}{8}} x^{2}}}\right)
$$

has maximum error less than that of the quantity

$$
0.5\left(1+\sqrt{1-e^{-\frac{2}{\pi} x^{2}}}\right)
$$

Now, let

$$
\Phi_{5}(x)=0.5\left(1+\sqrt{1-e^{-\frac{5}{8} x^{2}}}\right)
$$

be the proposed approximation for $\Phi(x)$. Because

$$
\frac{5}{8} \leq \sqrt{\frac{\pi}{8}} \leq \frac{2}{\pi}
$$

then

$$
\sqrt{1-e^{-\frac{5}{8} x^{2}}} \leq \sqrt{1-e^{-\sqrt{\frac{\pi}{8}} x^{2}}} \leq \sqrt{1-e^{-\frac{2}{\pi} x^{2}}} .
$$

This implies that

$$
\Phi_{5}(x) \leq \Phi_{2}(x) \leq \Phi_{1}(x) .
$$

Let $p=\Phi_{5}(x)$, then the approximation of the inverse function is

$$
x=\sqrt{-\frac{8}{5} \log \left(1-[2(p-0.5)]^{2}\right)} .
$$

To find the maximum error of $\Phi_{5}(x)$ as an approximation for $\Phi(x)$, define the function $h(x)$ as follows

$$
\begin{gathered}
h(x)=\Phi(x)-\Phi_{5}(x) \\
=\frac{1}{\sqrt{2 \pi}} \int_{0}^{x} e^{-\frac{t^{2}}{2}} d t-0.5 \sqrt{1-e^{-\frac{5}{8} x^{2}}}, x \geq 0 .
\end{gathered}
$$

The first derivative of $h(x)$ with respect to $x$ is $h^{\prime}(x)=\frac{e^{-\frac{x^{2}}{2}}}{\sqrt{2 \pi}}-\frac{0.3125 x e^{-\frac{5}{8} x^{2}}}{\sqrt{1-e^{-\frac{5}{8} x^{2}}}}$. By equating $h^{\prime}(x)$ with zero and solving with respect to $x$, we obtain the two roots

$$
x=0.56716111376 \text { and } x=1.91907381677 \text {. }
$$

The values of $h(x)$ at $x=0.56716111376$ and at $x=1.91907381677$ are 0.001317556983 and -0.001807859471 respectively. Also, the function $h^{\prime}(x)$ has a positive sign on $] 0,0.56716[\mathrm{U}] 1.91907, \infty[$ and negative sign on $] 0.56716,1.91907[$. Therefore, the extreme values of $h(x)$ occurs at $x=0.56716111376$ and at $x=1.91907381677$. This indicates that the absolute maximum error of $\Phi_{5}(x)$ is 0.00180786 . By comparing this maximum error with that of the others approximations, we conclude that the absolute maximum error of $\Phi_{5}(x)$ is less than the absolute maximum error of $\Phi_{1}(x), \Phi_{2}(x), \Phi_{3}(x)$ and $\Phi_{4}(x)$.

\section{Comparison}

In this section, we compare our approximation $\Phi_{5}(x)$ with the others one-term approximations $\Phi_{1}(x), \Phi_{2}(x)$, $\Phi_{3}(x)$ and $\Phi_{4}(x)$. We make this comparison for the values of $x=0.0$ to 4.0 with step 0.2 . The values of the different approximations are computed by using Wolfram Mathematica, 7, which are presented in Table (1). The corresponding errors between $\Phi(x)$ and the different approximations are also provided for purpose of simplicity comparison. From Table (1), we see that our approximation $\Phi_{5}(x)$ is the best one for $1.2 \leq x \leq 3.2$. In this case, the corresponding absolute error of $\Phi_{5}(x)$ is less than that of the other approximations. 
Table 1. The exact $\Phi(x)$ and the approximations $\Phi_{1}(x), \Phi_{2}(x), \Phi_{3}(x), \Phi_{4}(x)$ and $\Phi_{5}(x)$ of $\Phi(x)$. The differences between $\Phi(x)$ and the other approximations are given in the parentheses.

\begin{tabular}{|c|c|c|c|c|c|c|}
\hline$X$ & $\begin{array}{l}\text { Normal } \\
\Phi(\mathrm{x})\end{array}$ & $\begin{array}{l}\text { Polya } \\
\Phi_{1}(\mathrm{x}) \\
\end{array}$ & $\begin{array}{l}\text { Aludaat } \\
\Phi_{2}(\mathrm{x})\end{array}$ & $\begin{array}{c}\text { Bowling } \\
\Phi_{3}(\mathrm{x})\end{array}$ & $\begin{array}{c}\text { Lin } \\
\Phi_{4}(\mathrm{x})\end{array}$ & $\begin{array}{l}\text { Eidous } \\
\Phi_{5}(\mathrm{x})\end{array}$ \\
\hline \multirow[t]{2}{*}{0.0} & 0.5 & 0.5 & 0.5 & 0.5 & 0.5 & 0.5 \\
\hline & & (0) & (0) & (0) & (0) & (0) \\
\hline \multirow[t]{2}{*}{0.2} & 0.579260 & 0.57928 & 0.578668 & 0.584288 & 0.570918 & 0.578565 \\
\hline & & $(-0.00002)$ & $(0.000592)$ & $(-0.005028)$ & $(0.008341)$ & $(0.000694)$ \\
\hline \multirow[t]{2}{*}{0.4} & 0.655422 & 0.65560 & 0.654436 & 0.663917 & 0.642098 & 0.654242 \\
\hline & & $(-0.00018)$ & $(0.000985)$ & $(-0.008495)$ & $(0.013324)$ & $(0.001180)$ \\
\hline \multirow[t]{2}{*}{0.6} & 0.725747 & 0.72628 & 0.724700 & 0.735206 & 0.710456 & 0.724435 \\
\hline & & $(-0.00054)$ & $(0.001047)$ & $(-0.009459)$ & $(0.015291)$ & $(0.001312)$ \\
\hline \multirow[t]{2}{*}{0.8} & 0.788145 & 0.78924 & 0.787398 & 0.796020 & 0.773115 & 0.787089 \\
\hline & & $(-0.00110)$ & $(0.000747)$ & $(-0.007875)$ & $(0.015029)$ & $(0.001056)$ \\
\hline \multirow[t]{2}{*}{1.0} & 0.841345 & 0.84312 & 0.841184 & 0.845796 & 0.827897 & 0.840859 \\
\hline & & $(-0.00177)$ & $(0.000161)$ & $(-0.004451)$ & $(0.013448)$ & $(0.000486)$ \\
\hline \multirow[t]{2}{*}{1.2} & 0.884930 & 0.88736 & 0.885486 & 0.885177 & 0.873613 & 0.885172 \\
\hline & & $(-0.00243)$ & $(-0.000556)$ & $(-0.000247)$ & $(0.011317)$ & $(-0.000242)$ \\
\hline \multirow[t]{2}{*}{1.4} & 0.919243 & 0.92215 & 0.920474 & 0.915506 & 0.910100 & 0.920191 \\
\hline & & $(-0.00291)$ & $(-0.001231)$ & $(0.003737)$ & $(0.009143)$ & $(-0.000948)$ \\
\hline \multirow[t]{2}{*}{1.6} & 0.945201 & 0.94834 & 0.946922 & 0.938382 & 0.938025 & 0.946683 \\
\hline & & $(-0.00314)$ & $(-0.001722)$ & $(0.006819)$ & $(0.007175)$ & $(-0.001482)$ \\
\hline \multirow[t]{2}{*}{1.8} & 0.964070 & 0.96714 & 0.966024 & 0.955366 & 0.958576 & 0.965834 \\
\hline & & $(-0.00307)$ & $(-0.001954)$ & $(0.008704)$ & $(0.005494)$ & $(-0.001765)$ \\
\hline \multirow[t]{2}{*}{2.0} & 0.977250 & 0.98001 & 0.979181 & 0.967829 & 0.973153 & 0.979039 \\
\hline & & $(-0.00276)$ & $(-0.001931)$ & $(0.009421)$ & $(0.004097)$ & $(-0.001790)$ \\
\hline \multirow[t]{2}{*}{2.2} & 0.986097 & 0.98839 & 0.987809 & 0.976897 & 0.983136 & 0.987709 \\
\hline & & $(-0.00229)$ & $(-0.001712)$ & $(0.009200)$ & $(0.002960)$ & $(-0.001613)$ \\
\hline \multirow[t]{2}{*}{2.4} & 0.991802 & 0.99357 & 0.993188 & 0.983452 & 0.989744 & 0.993122 \\
\hline & & $(-0.00177)$ & $(-0.001385)$ & $(0.008351)$ & $(0.002058)$ & $(-0.001319)$ \\
\hline \multirow[t]{2}{*}{2.6} & 0.995339 & 0.99661 & 0.996371 & 0.988170 & 0.993971 & 0.996330 \\
\hline & & $(-0.00127)$ & $(-0.001032)$ & $(0.007169)$ & $(0.001368)$ & $(-0.000991)$ \\
\hline \multirow[t]{2}{*}{2.8} & 0.997445 & 0.99830 & 0.998159 & 0.991554 & 0.996582 & 0.998135 \\
\hline & & $(-0.00085)$ & $(-0.000714)$ & $(0.005891)$ & $(0.000863)$ & $(-0.000690)$ \\
\hline \multirow[t]{2}{*}{3.0} & 0.998650 & 0.99919 & 0.999111 & 0.993976 & 0.998136 & 0.999098 \\
\hline & & $(-0.00054)$ & $(-0.000461)$ & $(0.004674)$ & $(0.000514)$ & $(-0.000447)$ \\
\hline \multirow[t]{2}{*}{3.2} & 0.999313 & 0.99963 & 0.999591 & 0.995707 & 0.999026 & 0.999584 \\
\hline & & $(-0.00032)$ & $(-0.000279)$ & $(0.003606)$ & $(0.000287)$ & $(-0.000272)$ \\
\hline \multirow[t]{2}{*}{3.4} & 0.999663 & 0.99984 & 0.999821 & 0.996942 & 0.999514 & 0.999818 \\
\hline & & $(-0.00018)$ & $(-0.000158)$ & $(0.002721)$ & $(0.000149)$ & $(-0.000155)$ \\
\hline \multirow[t]{2}{*}{3.6} & 0.999841 & 0.99994 & 0.999926 & 0.997822 & 0.999770 & 0.999924 \\
\hline & & $(-0.00009)$ & $(-0.000085)$ & $(0.002019)$ & $(0.000071)$ & $(-0.000083)$ \\
\hline \multirow[t]{2}{*}{3.8} & 0.999928 & 0.99998 & 0.999971 & 0.998449 & 0.999897 & 0.999970 \\
\hline & & $(-0.00005)$ & $(-0.000043)$ & $(0.001478)$ & $(0.000030)$ & $(-0.000042)$ \\
\hline \multirow[t]{2}{*}{4.0} & 0.999968 & 0.99999 & 0.999989 & 0.998896 & 0.999957 & 0.999989 \\
\hline & & $(-0.00002)$ & $(-0.000021)$ & $(0.001072)$ & $(0.000011)$ & $(-0.000020)$ \\
\hline
\end{tabular}




\section{REFERENCES}

[1] Aludaat, K. M. and Alodat, M. T. (2008). A Note on Approximating the normal distribution function. Applied Mathematical science, 2 (9), 425-429.

[2] Johnson, N. I., Kotz, S. and Balakrishnan, N. (1994). Continuous univariate distributions. John Wiley \& Sons.

[3] Bailey, B. J. (1981). Alternatives to hasting's approximation to the inverse of the normal cumulative distribution function. Applied statistics, 30, (3) 275-276.
[4] Polya, G. (1945). Remarks on computing the probability integral in one and two dimensions. Proceeding of the first Berkeley symposium on mathematicalstatistics and probability, 63-78.

[5] Bowling, S. R., Khasawneh, M. T., Kaewkuekool, S. and Cho, B. R. (2009). A Logistic approximation to the cumulative normal distribution. Journal of Industrial Engineering and Management, 2(1), 114-127.

[6] Lin, J. T. (1990). A simple Logistic approximation to the normal tail probability and its inverse. Applied Statistics, 39, 255-257.

[7] Yerukala, R. and Boiroju N.K. (2015). Approximations to Standard Normal Distribution Function. International Journal of Scientific \& Engineering Research, 6(4),515-518. 